\title{
Assessment of chosen GRACE-related gravity models based on the GOCE satellite precise science orbit
}

\author{
Andrzej Bobojćc ${ }^{1}$ (D)
}

Received: 3 January 2019 / Accepted: 7 June 2019 / Published online: 18 June 2019

(c) The Author(s) 2019

\begin{abstract}
Eight selected geopotential models obtained through the International Center for Global Earth Models were used in the dynamic orbit determination process of the satellite of the Gravity Field and Steady-State Ocean Circulation Explorer (GOCE) mission. For the estimation of various GOCE orbital arc variants, the following gravity models were taken into account: HUST-GRACE2016S, ITU_GRACE16, ITSG-GRACE2014S, ITSG-GRACE2014K, TONGJI-GRACE01, EIGEN-51C, EIGEN5S, EGM2008. The official kinematic and reduced-dynamic precise science orbit (PSO) of the GOCE satellite received via the European Space Agency was adopted as the reference orbit. Cartesian coordinates of the GOCE satellite in this orbit were treated as pseudo-observations in the estimation process using the classical least-squares method. The estimated orbital arcs were fitted to the corresponding arcs of the reference orbit. This allowed the values of 3D root-mean-square (RMS) of the distance between the estimated and reference arcs to be computed. The averages for these 3D RMS values, computed for ten and fifty orbital arcs, made it possible to compare the orbital performance of selected gravity models. Additionally, the fit to both types of the GOCE PSO, i.e., the kinematic orbit and the reduced-dynamic orbit made it possible to compare their quality. Investigation of the gravity model performance was also the opportunity to describe the effectiveness of dynamic orbit determination solutions, depending on the estimated arc lengths, type of reference orbit and the use (or not) of the background models.
\end{abstract}

Keywords GRACE gravity models · GOCE Precise Science Orbit · Dynamic orbit determination

\section{Introduction}

Among the many factors determining processes in the Earth's interior and exterior, the gravity field is of key importance. An accurate knowledge of the gravity field is also crucial for satellite orbits, which serve for precise geolocation of various measurements conducted in satellite missions, for example, for such missions as: the Challenging Mini-Satellite Payload (CHAMP) (Reigber et al. 2005), the Gravity Recovery and Climate Experiment (GRACE) (Tapley et al. 2004) and the Gravity Field and Steady-State Ocean Circulation Explorer (GOCE) (Rummel et al. 2011). Therefore, in recent years, the gravity field recovery was the subject of numerous works leading to many gravity field models. These models are mostly connected with the three

Andrzej Bobojć

altair@uwm.edu.pl

1 Institute of Geodesy, University of Warmia and Mazury in Olsztyn, Olsztyn, Poland aforementioned satellite missions. The estimated gravity models were validated and compared with each other, taking into account different aspects of their performance. Generally, these aspects can be divided into two main test areas: The first area is related to the Earth itself and the second area to satellite orbits. Concerning the first test area, for example, Gruber et al. (2011) assessed gravity models comparing the geoid heights obtained from the given gravity models with the corresponding ones estimated from the GPS levelling. Various authors compared the GOCE-related gravity models in terms of the gravity anomaly and the geoid height differences in local areas such as Germany (Voigt and Denker 2015), Norway (Šprlák et al. 2015), the Mediterranean area (Carrion et al. 2015) and on the continental scale —in South America (Matos et al. 2015). In turn, Hirt et al. (2015) evaluated the GOCE gravity models by comparing them with gravity models derived from the ET2014 topography. Yi and Rummel (2014) compared the GOCE-related gravity models with the EGM2008 model. This comparison was performed globally as well as in selected geographical regions using 
geoid root-mean-square (RMS) differences. Xu et al. (2017) compiled the GOCE-only gravity model GOSG01 and investigated its consistency with other GOCE-related satellite models, taking into account GPS-levelling data.

In the aforementioned second test area, Lejba et al. (2007), Sośnica et al. (2012) and Cheng and Ries (2015), taking into account the satellite laser ranging (SLR) observations, investigated the RMS of SLR residuals coming from the estimation of the Laser Geodynamics Satellite (LAGEOS) orbits using given gravity models. The three GOCE-related gravity models were tested by Gruber et al. (2011), who compared orbital residuals derived in the frame of orbit determination of the CHAMP satellite and both GRACE satellites. Tsoulis and Papanikolaou (2012) computed short arcs of the CHAMP and GRACE-A satellites using a numerical integration method and compared them with the corresponding Rapid Science Orbit (RSO) for different tested geopotential models. The authors indicated the band-limited behavior of gravity models in an orbit determination process. In their next two works, Tsoulis and Papanikolaou $(2013,2014)$ validated GRACE and CHAMP mission-related gravity models by the short-arc GOCE orbit propagation using the GOCE Rapid Science Orbit data as a reference and tested the GOCE-related gravity models fitting the adjusted daily GOCE orbits into positions in the precise science orbit (PSO) (Bock et al. 2011) and in the GRACE-A and GRACE-B satellite precise orbits. In these works, the authors emphasize the spectral contribution of individual gravity models in a degree-wise cumulative sense. Förste et al. (2014) described the performance of the high-degree EIGEN-6C4p model comparing the estimated GOCE orbits to the PSO. Baur et al. (2014) compared the relative performance of GOCE gravity field models which resulted in a comparison of different estimation approaches of these models. The adopted gravity fields were estimated using the GOCE kinematic orbits.

This work, through entering the trend of the aforementioned research, focuses on an evaluation of the eight chosen GRACE-related gravity models in terms of the dynamic orbit determination, by fitting obtained positions into the positions taken from the official PSO, consisting of the two types of orbits: kinematic orbits and reduced-dynamic orbits, which were delivered by the European Space Agency (ESA). Both types of GOCE orbit were validated using the satellite laser ranging (SLR) technique by means of residuals determined as differences between ranges based on SLR and ranges derived from the aforementioned kinematic and reduced-dynamic orbits (Strugarek et al. 2018). Finally, SLR validation results for the entire GOCE mission allowed an accuracy of $2.42 \mathrm{~cm}$ to be determined for the kinematic orbit and $1.84 \mathrm{~cm}$ for the reduced-dynamic orbit (Bock et al. 2014). However, taking into account the time range of the used GOCE orbits, these accuracies were slightly different in the years 2009 and $2010-1.91 \mathrm{~cm}, 1.84 \mathrm{~cm}$ for the kinematic orbit and $1.73 \mathrm{~cm}, 1.56 \mathrm{~cm}$ for the reduced-dynamic orbit (Bock et al. 2014).

The kinematic orbit is a purely geometric solution based on the satellite-to-satellite tracking (SST) measurements (Bock et al. 2011), whereas the reduced-dynamic orbit is derived by the reduced-dynamic approach, in which, besides the SST measurements, the a priori gravity model and additional dynamic models are used. Moreover, the dynamic part of this approach is limited by the estimation of set empirical parameters - in this case, pseudo-stochastic, piece-wise constant accelerations (Bock et al. 2011). The fitting of the estimated orbits to the kinematic orbit is motivated by independence of the GOCE kinematic orbit from any background dynamic models, especially from any a priori gravity model. On the other hand, the fit to the reduced-dynamic orbit was also performed because of its higher smoothness and greater resistance to measurement problems. The use of both GOCE orbit types to the fitting of estimated arcs was also an opportunity to compare the quality of these orbits. The GOCE orbit is particularly suitable for the presented study because of its extremely low altitude at about $255 \mathrm{~km}$, where the gravitational signal is as large as possible (Tsoulis and Papanikolaou 2013). On the other hand, the selection of gravity models not related to the GOCE mission avoids possible correlations via the underlying SST measurements. Therefore, the gravity models derived from the observational data coming from the GOCE mission are not included in this study.

It is well known that the gravity field model is a key component in a satellite orbit determination process, strictly speaking in the dynamic orbit determination. However, existing numerous gravity models require an appropriate selection for aforementioned process. The gravity models being the products of the GRACE mission are known for their good quality and constitute a large group in the entire collection of available models (Drewes 2012). Therefore, this work focuses on comparing an orbital performance of the selected gravity models coming from the mentioned group. Due to the fact that all tested models come from the same mission, showing differences in their performance in an orbital aspect may be a demanding task. In order to reveal these differences, different variants of computations, including different numbers of orbital arcs, their different lengths and using background models or not, were adopted in the work. Finding these differences in the orbital performance and possible patterns associated with them is one of the goals of this work. The basic question is whether it is possible, and to what extent, to determine statistically significant differences in the performance of selected gravity models in an orbital aspect? Another purpose of the work resulting from the aforementioned goal may be the possibility of using the obtained numerical results to the aforementioned 
selection of gravity field models for the dynamic orbit determination process of a low Earth orbiting satellite-for example, the fully dynamic orbit determination of the GOCE satellite, as a low Earth orbiting satellite, was performed by Casotto et al. (2013). The centimeter-accuracy PSO GOCE satellite orbit, as an example of low Earth orbiter trajectory, can be a good reference in the context of selection of appropriate gravity models for a description of motion of a satellite orbiting at a low altitude. On the other hand, as mentioned above, the use of such a low satellite as GOCE for the research is particularly convenient due to the weakening of the gravitational signal with increasing altitude, which causes the orbit to be sensitive only to a certain range of spherical harmonic coefficients. This limits the comparison of the gravity model performance only to the long-wavelength parts. In the case of GOCE satellite, the limitation of long-wavelength part of gravity model is equal to about the degree and order of 120 spherical harmonic coefficients. This limitation is indicated by Bezděk et al. (2014) and Baur et al. (2014), who obtained static gravity field models from the kinematic positions of various satellites, such as GRACE A/B, CHAMP and GOCE.

In "Investigation procedure" section of this work, a short description of the research procedure, including the dynamic orbit determination process, and the characteristics of investigated gravity models are given. The results of the orbit determination process for the three chosen arc lengths and a performance comparison of gravity models are presented and described in "Research results" section. Finally, "Summary and conclusions" section contains a summary and concluding remarks with the highlighted performance differences for the selected gravity models.

\section{Investigation procedure}

All computations were performed using a software package called the Torun Orbit Processor (TOP). One of the first versions of this software was presented in Drożyner (1995). The TOP package is based on the dynamic orbit determination algorithm, where a satellite orbit is integrated numerically using the Cowell 8 th-order predictor-corrector component. The satellite orbital arcs obtained, including gravitational and non-gravitational perturbing forces, are in the form of a time series of state vectors consisting of position and velocity of the satellite. Additionally, the satellite orbit can be improved by incorporating a set of observations. As already mentioned, centimeter-level accuracy Cartesian coordinates (Bock et al. 2011) of the GOCE satellite along the kinematic and reduced-dynamic orbits were treated here as observations. These coordinates, originally expressed in the ITRF2005 reference frame, were transformed into the inertial reference frame (IRF) connected with the standard epoch J2000.0 (ESA 2010). This was possible by using a set of the ESA-delivered quaternions (ESA 2010) which described the transformation between both reference frames.

The GOCE orbital arcs were estimated in an iterative process using the least-squares method. In this process, the aforementioned quaternions were also used. Besides gravity models, a set of additional force models was optionally used. These background force models can be divided into two groups: The first one describes gravitational perturbing forces and the second one describes non-gravitational perturbing forces (Eshagh and Najafi-Alamdari 2007). In this case, the following additional forces were modeled: ocean tides and Earth tides-the Monitoring Earth Rotation and Intercomparison of Techniques (MERIT) standards model (Melbourne et al. 1983), third body effectsthe planetary ephemerides DE200/LE200 (Standish et al. 1992), relativity effects - the Painleve formulation, taking into account spherical symmetrical space-time with the Schwarzschild metric, solar radiation pressure (direct and albedo effects) - models implemented in the TOP package (Drożyner 1995). The air drag pressure effect is omitted here due to its compensation in the drag-free mode flight of the GOCE satellite (Bock et al. 2011).

The computed orbital arcs were parametrized by components of the satellite initial state vector. Strictly speaking, the estimated unknowns were six corrections to the mentioned components, i.e., to the initial position and velocity. Therefore, the observation equation can be written in the form:

$x_{i k}^{o}+v_{i k}=x_{i k}^{c}+\frac{\partial x_{i k}^{c}}{\partial \boldsymbol{p}_{0}} \Delta \boldsymbol{p}_{0}^{\mathrm{T}}$.

In this equation, $x_{i k}^{o}, x_{i k}^{c}(i=1,2,3)$ are the observed and computed $i$-th coordinate of the satellite at the $k$-th epoch, respectively, $v_{i k}$ is the correction to the observed $i$-th coordinate at the $k$-th epoch, $\boldsymbol{p}_{0}$ is the initial state vector and $\Delta \boldsymbol{p}_{0}$ is the estimated correction vector to the initial state vector $\boldsymbol{p}_{0}$. The numerical integration of the variational equations in the orbit improvement process makes it possible to determine the partial derivatives $\partial x_{i k}^{c} / \partial \boldsymbol{p}_{0}$ at the $k$ -th epoch in the Eq. (1).

The use of simplified parameterization in the adjustment process is motivated by the need to emphasize the contribution of the given gravity models to the orbit determination. In another case, additional estimated empirical parameters could absorb some part of the gravity model error (Tsoulis and Papanikolaou 2014). To further increase the gravity field model contribution, some variants of computations were performed without the background force models. On the other hand, the computation variants, 
including the background force models, made it possible to assess the degree of dependence of the obtained results on the specified set of these models. The taken lengths of determined arcs are equal to 45.2, $90.2 \mathrm{~min}$ and 1 day, which is close to the half, the one and near the sixteen GOCE revolution periods, respectively. The selection of the three different arc lengths is motivated by the need to investigate the question: To what extent do the differences of performance of the specified gravity models change when the length of the estimated orbital arc changes?

The obtained orbits for the specified gravity models were compared to the corresponding GOCE kinematic and reduced-dynamic orbits in the IRF. As a result, for the comparison of the performance of gravity models, the 3D RMS differences between both orbits were computed. These RMS values are based on the differences between the estimated and observed kinematic and reduced-dynamic positions of the GOCE satellite. The estimated arcs were included in the time range spanning between November 02, 2009, and February 11, 2010. The approximate values of the initial positions and velocities were adopted from both reference orbits, i.e., from the kinematic and reduced-dynamic orbits. The fifty initial epochs have been assigned to the fifty estimated arcs with the following dates: in 2009-November 02 to November 25, November 28 to December 02, December 18 , December 29; in 2010 - January 06, January 08 to January 09 , January 12 to January 16, January 18 to January 26, February 05, February 10. For all dates, except February 05, 2010 , the same moment of time ( $23 \mathrm{~h} 59 \mathrm{~m} 45.00 \mathrm{~s}$ UTC) is assigned. For February 05, 2010, this moment is $23 \mathrm{~h} 29 \mathrm{~m}$ $55.22 \mathrm{~s}$ UTC.

In Table 1, the GRACE-related gravity models taken for testing have been specified. Thanks to the International Center for Global Earth Models (ICGEM) at Deutsches GeoForschungsZentrum Potsdam (Drewes 2012), these models were obtained as sets of normalized dimensionless coefficients of spherical harmonic expansion. In this study, the EGM2008 gravity model was truncated at degree and order 360. For each model, the corresponding types of data used in its estimation and the data period are also given. Taking into account the data period, the chosen gravity models can be divided into two groups. The first group of older models for which the data used is from before 2010. These are the first four models from Table 1, i.e., EIGEN-5S, EIGEN-51C, EGM2008 and TONGJI-GRACE01. The second group of newer models, with the part of data acquired after 2010, includes the next four models: ITSG-GRACE2014K, ITSGGRACE2014S, ITU_GRACE16 and HUST-GRACE 2016S.

\section{Research results}

As mentioned above, taking into account the positions from the estimated orbital arcs and the GOCE kinematic and reduced-dynamic positions, the resulting set of RMS values was obtained (being a measure of fit of the mentioned arcs to the corresponding GOCE kinematic and reduced-dynamic arcs). Several computation variants were obtained. In each variant, a set of the ten or fifty RMS values corresponding to the fit of the estimated arcs to the ten or fifty selected GOCE reference arcs was determined for the given gravity model. Thus, the performance comparison of the selected gravity models was based on the averaged RMS values. Below, these averaged RMS values will be marked simply as RMS.

Generally, two basic computational variants were realized. In the first basic variant, the gravity models were tested in terms of the fit to the ten aforementioned short arcs of kinematic and reduced-dynamic orbits with lengths 45.2 and $90.2 \mathrm{~min}$. For both arc lengths, the maximum differences between RMS values (not shown here) for the particular gravity models do not exceed $0.4 \mathrm{~cm}$ for the arc length of $45.2 \mathrm{~min}$ and $1.3 \mathrm{~cm}$ for the arc length of $90.2 \mathrm{~min}$. Taking into account the accuracy of determining the GOCE PSO at the level of $2 \mathrm{~cm}$, this means that the obtained differences of RMS values do not have statistical significance. In other words, the performance of all tested gravity models is the same within the accuracy of the GOCE PSO. However, the obtained RMS values averaged for all gravity models and the estimated ten orbital arcs can be compared for both lengths of arcs and for both types of the PSO when the background models are switched on and off. These RMS values (as averages of eighty values) are presented in Table 2 . It is clearly
Table 1 Gravity models investigated in this work and data used in their recovery

\begin{tabular}{lll}
\hline Gravity model & Kinds of data and data period & References \\
\hline EIGEN-5S & GRACE, LAGEOS, 2002-2007 & Förste et al. (2008) \\
EIGEN-51C & GRACE, CHAMP, altimetry terrestrial data, 2002-2008 & Bruinsma et al. (2010) \\
EGM2008 & GRACE, altimetry, terrestrial data, 2002-2007 & Pavlis et al. (2012) \\
TONGJI-GRACE01 & GRACE, 2003-2007 & Chen et al. (2015) \\
ITSG-GRACE2014K & GRACE, 2003-2013 & Mayer-Gürr et al. (2014) \\
ITSG-GRACE2014S & GRACE, 2003-2013 & Kvas et al. (2014) \\
ITU_GRACE16 & GRACE, 2009-2013 & Guo et al. (2015) \\
HUST-GRACE 2016S & GRACE, 2003-2016 & Zhou et al. (2017) \\
\hline
\end{tabular}


Table 2 RMS values averaged for all compared gravity models and the ten estimated orbital arcs depending on the computation variant with the different estimated arc lengths $-45.2 \mathrm{~min}$ and $90.2 \mathrm{~min}$, the different reference orbits and with or without switching the background models

\begin{tabular}{|c|c|c|c|}
\hline \multirow[t]{3}{*}{ Reference } & \multicolumn{2}{|l|}{ RMS [cm] } & \multirow{3}{*}{$\begin{array}{l}\text { Ratio }^{\mathrm{b}} \\
(\operatorname{arc} 1) /(\operatorname{arc} 2)\end{array}$} \\
\hline & $45.2 \min (\operatorname{arc} 1)$ & $90.2 \min (\operatorname{arc} 2)$ & \\
\hline & $\mathrm{BM}$ off/BM on ${ }^{\mathrm{a}}$ & $\mathrm{BM}$ off/BM on & \\
\hline Kinematic PSO & $22.75 / 5.15$ & $53.37 / 13.50$ & $4.42 / 3.95$ \\
\hline \multirow{2}{*}{$\begin{array}{l}\text { Reduced-dynamic } \\
\text { PSO }\end{array}$} & $22.71 / 4.82$ & $53.75 / 13.08$ & $4.71 / 4.11$ \\
\hline & & Mean ratio: & $4.56 / 4.03$ \\
\hline
\end{tabular}

${ }^{\mathrm{a}} \mathrm{BM}$ off/BM on-background models (BM) on/off in the orbit determination process

${ }^{\mathrm{b}} \mathrm{RMS}$ without the BM to RMS with the BM ratio

seen that the twofold increase in the arc length in all cases is associated with more than a twofold increase in the RMS value.

But what is characteristic for the variants without taking into account the background models (BM), the RMS values increase by about a factor of near 2.3, but while taking into account the BM, these values increase to about a factor of 2.6. On the other hand, the ratios obtained by dividing RMS values without the BM by RMS values with the BM are also shown in Table 2. These ratios can be treated as a measure of the relative effectiveness of using the BM. As one can see, the ratio values depend both on the arc length and on the type of the used reference orbit. The use of the BM is more effective for the shorter $45.2 \mathrm{~min}$ arc (ratios 4.42, 4.71) than for the longer $90.2 \mathrm{~min}$ arc (ratios 3.95, 4.11) and is also more effective for the reduced-dynamic PSO (RD) as the reference (ratios 4.71, 4.11) than for the kinematic PSO (KIN) as the reference (ratios 4.42, 3.95). The decrease in efficiency of the performance of the BM at the increasing length of the estimated arc may be related to the growing systematic error caused by the simplified parameterization of the orbit. In turn, the higher efficiency of the BM in the case of the fit to the RD orbit than to the KIN orbit is connected with a greater smoothness of the RD orbit compared to the KIN orbit due to the existence of a dynamic part in the process of the RD orbit determination (Bock et al. 2014). However, for the above first basic computational variant, the fit to the KIN orbits and to the RD orbits was associated with a 1-s and 10-s sampling interval, respectively. The higher sampling interval for the RD orbits may be the additional factor determining the better fit to these orbits.

As already mentioned above, the maximum differences between the computed RMS values for the particular gravity models are equal to $0.4 \mathrm{~cm}$ and $1.3 \mathrm{~cm}$ for the arc length of $45.2 \mathrm{~min}(\operatorname{arc} 1)$ and of $90.2 \mathrm{~min}(\operatorname{arc} 2)$, respectively. This indicates that these differences increase with the growing arc length. Therefore, in order to obtain the statistical significance of RMS values, the second basic variant of computation was performed. This variant includes the estimation of 1-day arcs with respect to both reference orbit types, i.e., the fit of determined 1-day arcs to the corresponding 1-day arcs of the KIN and RD orbits. For the fit to the 1-day reference orbits, a 60-s sampling interval was adopted. The results are presented in Tables 3 and 4 as RMS values computed as averages for estimated arcs fitted to the corresponding KIN and RD reference arcs. For the KIN reference orbit, RMS values were computed, taking into account the corresponding values for the nine estimated arcs. The value of RMS for the fit to the 1-day KIN reference arc with the initial epoch

Table 3 RMS values of the fit to the 1-day KIN orbits as averages of nine values for each investigated gravity model ${ }^{\mathrm{a}}$

\begin{tabular}{llll}
\hline Gravity model & $\begin{array}{l}\text { RMS (m) } \\
\text { BM off }\end{array}$ & Gravity model & $\begin{array}{l}\text { RMS (m) } \\
\text { BM on }\end{array}$ \\
\hline EIGEN-5S & 6.31 & TONGJI-GRACE01 & 4.52 \\
HUST-GRACE2016S & 6.37 & HUST-GRACE2016S & 4.53 \\
TONGJI-GRACE01 & 6.37 & EIGEN-5S & 4.54 \\
ITSG-GRACE2014K & 6.58 & ITSG-GRACE2014K & 4.65 \\
EIGEN-51C & 6.67 & EIGEN-51C & 4.95 \\
EGM2008 & 6.71 & EGM2008 & 5.06 \\
ITU-GRACE16 & 7.16 & ITU-GRACE16 & 5.51 \\
ITSG-GRACE2014S & 7.45 & ITSG-GRACE2014S & 5.80 \\
Mean RMS [m] & 6.70 & & 4.94 \\
Ratio of mean RMS & & 1.36 & \\
\hline
\end{tabular}

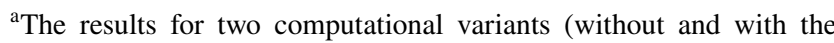
$\mathrm{BM})$ are given

${ }^{\mathrm{b}}$ Mean RMS for the BM off divided by the mean RMS for the BM on

Table 4 RMS values of the fit to the 1-day RD orbits as averages of ten values for each investigated gravity model $^{\mathrm{a}}$

\begin{tabular}{llll}
\hline Gravity model & $\begin{array}{l}\text { RMS (m) } \\
\text { BM off }\end{array}$ & Gravity model & $\begin{array}{l}\text { RMS (m) } \\
\text { BM on }\end{array}$ \\
\hline EIGEN-5S & 7.04 & TONGJI-GRACE01 & 2.08 \\
HUST-GRACE2016S & 7.14 & HUST-GRACE2016S & 2.09 \\
TONGJI-GRACE01 & 7.14 & EIGEN-5S & 2.10 \\
EIGEN-51C & 7.39 & ITSG-GRACE2014K & 2.75 \\
EGM2008 & 7.46 & EIGEN-51C & 2.86 \\
ITSG-GRACE2014K & 7.52 & EGM2008 & 2.94 \\
ITU-GRACE16 & 7.93 & ITU-GRACE16 & 3.54 \\
ITSG-GRACE2014S & 8.29 & ITSG-GRACE2014S & 4.19 \\
Mean RMS [m] & 7.49 & & 2.82 \\
Ratio of mean RMS & & 2.65 & \\
\hline
\end{tabular}

${ }^{a}$ The results for two computational variants (without and with the BM) are presented 
Dec $1823 \mathrm{~h} 59 \mathrm{~m} 45.00 \mathrm{~s}$ UTC was at a level of $250 \mathrm{~m}$ due to data gaps-missing kinematic positions for a number of epochs.

Thus, the results for this arc can be treated in some sense as outliers. Comparing these results to the results for the short arcs, it is clearly seen that RMS values increased from the level of centimeters and decimeters to the level of meters. Along with this increase, there was also an increase in the difference between the RMS values for the particular gravity models. These differences in the fit to the 1-day reference orbits reach over two meters, significantly exceeding the two-centimeter threshold associated with the accuracy of the reference orbits. Looking at the mean RMS values in Tables 3 and 4 for variants without using the BM, it can be seen that, similar to the case of the $90.2 \mathrm{~min}$ arc, the better fit of estimated arcs is achieved for the KIN orbit (mean RMS $6.70 \mathrm{~m}$ ) than for the RD orbit (mean RMS $7.49 \mathrm{~m}$ ). It seems that this may be connected with a greater level of the distance of estimated arcs with respect to the reference orbit without using the BM. However, enabling the BM, as for the short arcs, gives a better fit to the RD orbit-the mean RMS $2.82 \mathrm{~m}$ against $4.94 \mathrm{~m}$ for the KIN orbit.

This is reflected by the ratio of mean RMS values 2.65 for the fit to the RD orbit, which is, similarly as for the short arcs, clearly larger than the corresponding value of 1.36 for the fit to the KIN orbit. Thus, the greater efficiency in the use of $\mathrm{BM}$ in terms of the fit to the RD orbits is also confirmed for the longer 1-day arcs. On the other hand, taking into account the increasing orbital arcs, it is clearly seen that, in this aspect, the aforementioned efficiency decreases-the mean ratio is 4.56 and 4.03 (Table 2) for the $45.2 \mathrm{~min}$ arcs and $90.2 \mathrm{~min}$ arcs, respectively, whereas this ratio is 2.00 (averaged the ratio of the mean RMS values for both types of the reference orbits from Tables 3 and 4) for the 1-day arcs. In general, the above results indicate the clearly better fit of the estimated orbital arcs to the RD orbit than to the KIN one. As already mentioned, this could be associated with a greater smoothness of the RD orbit than for the KIN orbit, which is strongly dependent on the quality of GPS measurements. This could confirm the greater accuracy of the RD orbit.

Essentially in Tables 3 and 4, the relative performance of the selected GRACE mission-related gravity models is given in terms of RMS of the fit of estimated arcs based on these models. The investigated models can be divided into three groups. The first one includes the gravity models such as TONGJI-GRACE01, HUST-GRACE2016S and EIGEN5S. These three models clearly dominate for all computational variants with smaller RMS values that differ from the RMS values for the remaining models by $11 \mathrm{~cm}$ to $65 \mathrm{~cm}$ (Tables 3 and 4). The characteristic pattern for this group can be noticed-in the computational variants for both reference orbits without the BM, the gravity model EIGEN-5S exhibits the best performance by $6 \mathrm{~cm}$ (Table 3 ) and $10 \mathrm{~cm}$ (Table 4). The two remaining models HUST-GRACE2016S and TONGJI-GRACE01 achieve the same RMS values for the fit to the KIN and RD orbits- $6.37 \mathrm{~m}$ and $7.14 \mathrm{~m}$, respectively. For the computational variants with the BM, the order of the gravity models is the same for the KIN and RD orbits: TONGJI-GRACE01, HUST-GRACE2016S, EIGEN-5S. However, the differences between RMS values are $2 \mathrm{~cm}$ for both computational variants (Tables 3 and 4). This means that these differences remain at a level of the reference orbit accuracy (Bock et al. 2014). In this case, it can be stated that the performance of these models is the same. Thus, the inclusion of the BM has led to the same performance of these three gravity models. The outstanding performance of EIGEN-5S is also not a surprise. This model truncated to the degree and order of 120 was the a priori gravity model in the estimation process of RD orbits (Bock et al. 2014). But at this point it is worth noting that this model also has outstanding performance in terms of the fit to the KIN orbits, which are directly independent of any dynamic models. In the case of the fit to the RD orbits without the BM, EIGEN-5S is the best-performing model (Table 4). This is especially important because the computational variant without using the BM is independent of the specific set of used BM and thus may be treated with a slightly greater weight than the variant using the given BM.

The second group of gravity models includes ITSGGRACE2014K, EIGEN-51C, EGM2008. It is worth noting, for the three computational variants (without and with using the BM with respect to the KIN reference orbit and with using the BM with respect to the RD reference orbit), that the order of these models according to increasing RMS values is the same-as listed above. In the fourth variant (without the BM with respect to the RD orbit), ITSGGRACE2014K moved to the third position while the order of the two remaining models is still the same, i.e., in this case, there is the following order: EIGEN-51C, EGM2008, ITSG-GRACE2014K (Table 4).

Finally, the third group of models with clearly higher RMS values includes ITU-GRACE16 and ITSG-GRACE2014S. The difference in RMS between the first of these models and the last model of the third group varies from $41 \mathrm{~cm}$ to $60 \mathrm{~cm}$ depending on the computational variant (Tables 3 and 4). As one can see, in all cases ITSG-GRACE2014S has the highest RMS values among all tested models. In comparing the performance of the two ITSG-GRACE2014-type gravity models, it is clearly seen that in all computational variants the regularized ITSG-GRACE2014K model (Mayer-Gürr et al. 2014) has the lower RMS values than the unconstrained ITSG-GRACE2014S model (Kvas et al. 2014).

Taking into account the results presented in Tables 3 and 4 for the computational variant without the use of the BM and for the variant with the use of the BM, one can see some 
Table 5 Ratio of RMS values for the selected gravity models depending on the reference orbit

\begin{tabular}{lll}
\hline Gravity model & \multicolumn{2}{l}{ Ratio of RMS } \\
\cline { 2 - 3 } & Reference KIN orbit & $\begin{array}{l}\text { Reference } \\
\text { RD orbit }\end{array}$ \\
\hline EIGEN-5S & 1.39 & 3.35 \\
HUST-GRACE2016S & 1.41 & 3.42 \\
TONGJI-GRACE01 & 1.41 & 3.43 \\
EGM2008 & 1.33 & 2.54 \\
ITU-GRACE16 & 1.30 & 2.24 \\
ITSG-GRACE2014S & 1.28 & 1.98 \\
\hline
\end{tabular}

Table 6 Growth rates of the RMS values for the fit of estimated arcs to the KIN and RD orbits

\begin{tabular}{|c|c|c|}
\hline \multirow[t]{3}{*}{ Arc length (min) } & \multicolumn{2}{|c|}{ RMS growth rate $(\mathrm{cm} / \mathrm{min})$} \\
\hline & Reference KIN orbit & Reference RD orbit \\
\hline & $\mathrm{BM}$ off/on ${ }^{\mathrm{a}}$ & BM off/on \\
\hline 45.2 & $0.50 / 0.11$ & $0.50 / 0.11$ \\
\hline 90.2 & $0.59 / 0.15$ & $0.60 / 0.14$ \\
\hline 1440 & $0.46 / 0.34$ & $0.52 / 0.20$ \\
\hline
\end{tabular}

${ }^{\mathrm{a}} \mathrm{BM}$ off/on in the orbit determination process

partial impact of using the given set of BM on the order of gravity models according to the increasing RMS values. On the one hand, the same three groups of models can also be indicated after enabling the BM, but on the other hand: In the first group - the performance of all three models became equal, and in the second group-a partial change in the order took place-ITSG-GRACE2014K "jumped" from the third to the first position in the group. Generally speaking, after applying the BM for the fit to both types of the reference orbits, there were no significant changes in the order of the gravity models - the basic division of gravity models into the three groups remained unchanged with the two minor aforementioned changes.

In order to illustrate the dependence of the effectiveness of using the BM on the selected gravity models, the ratios of the RMS values were computed by dividing RMS for variants without the BM by RMS for variants with the use of the BM (Table 5). These results were also calculated for both types of the reference orbits. It is clear that with the gravity models: EIGEN-5S, HUST-GRACE2016S, TONGJIGRACE01 (Tables 3 and 4), a higher effectiveness of using the $\mathrm{BM}$ in terms of the fit to both types of the reference orbits is connected. For the gravity models: EGM2008, ITUGRACE16, ITSG-GRACE2014S, the clearly lower ratios were obtained (Table 5).

In Table 6, the growth rates of RMS values are presented. These values were obtained by dividing the mean RMS for the fit to both KIN and RD orbits by the length of the corresponding arc. The growth rates of RMS were also separately calculated for the variants without and with the use of the BM.

From the different values of the RMS growth rates, the non-linear increase of RMS values (for both variants-without and with the BM) results. For variants without the BM, the RMS growth rates are clearly greater than in the case of the variants with the BM. But in the first case (without the $\mathrm{BM}$ ), the RMS growth rates do not show any trend remaining on average on the level of $0.53 \mathrm{~cm} / \mathrm{min}$, whereas in the second case (with the BM) the RMS growth rates clearly increase for both the KIN and RD orbits (Table 6). This increase confirms the aforementioned decrease in the effectiveness of using BM with an increase in the estimated arc length. Lower rate values for the RD orbits also confirm the better fit of the estimated arcs for these orbits than for the KIN orbits, especially in the computational variants using the BM.

So far, all the results were based on the average RMS values for the fit of ten arcs for each gravity model. To strengthen the statistics presented, the fit of fifty 1-day orbital arcs to the corresponding RD reference arcs was performed for each tested gravity field model. Additionally, the BM were taken into account in this computation. The obtained set of RMS values for the gravity models are given in Table 7. The listed dates correspond to the begin of initial epochs at the moment of time $23 \mathrm{~h} 59 \mathrm{~m} 45.00 \mathrm{~s}$ UTC. At the bottom of Table 7, the mean values of RMS with the standard deviations for all models are presented. The minimal and maximal value of RMS is also listed for each model. It is clearly seen that the differences between RMS values for all models are at the level of decimeters and meters significantly exceeding the accuracy level of the reference orbit. Comparing the mean RMS values in Table 4 (estimation of 10 orbital arcs per model) with the corresponding values in Table 7 (estimation of 50 arcs per model), it is evident that the increase in the number of statistic data did not significantly change the relative differences in the performance of individual gravity models, on the contrary, a very similar image emerges from both sets of results. First of all, the division of tested models into the three groups still exists. The first group includes: EIGEN-5S, HUST-GRACE2016S, TONGJI-GRACE01. This time in all three cases the mean RMS is exactly the same- $2.32 \mathrm{~m}$. The two models of second group, i.e., ITSG-GRACE2014K and EIGEN-51C, have the same mean RMS value- $2.52 \mathrm{~m}$, whereas the RMS value is slightly larger (2.57 m) for EGM2008, similarly as in Table 4 (for the ten arcs per model). Finally, the gravity models ITU-GRACE16 and ITSG-GRACE2014S belong to the third group with the largest RMS values $2.96 \mathrm{~m}$ and $3.37 \mathrm{~m}$, respectively. It is also noticeable that the differences in the performance of particular models are reduced when 
Table 7 RMS values of the fit to the fifty 1-day RD orbits (initial epochs from the range November 2, 2009-February $10,2010)$ for each investigated gravity model ${ }^{\mathrm{a}}$

\begin{tabular}{|c|c|c|c|c|c|c|c|c|}
\hline Date & $\begin{array}{l}\text { HUST- } \\
\text { GR. } \\
2016 S\end{array}$ & ITU-GR.16 & $\begin{array}{l}\text { ITSG- } \\
\text { GR.2014S }\end{array}$ & $\begin{array}{l}\text { ITSG- } \\
\text { GR.2014K }\end{array}$ & TON.-GR.01 & EIG.-51C & EIG.-5S & EGM2008 \\
\hline Nov 02 & 1.94 & 3.35 & 2.93 & 2.45 & 1.94 & 1.91 & 2.11 & 2.04 \\
\hline 3 & 2.01 & 2.02 & 5.69 & 2.02 & 2.00 & 1.66 & 1.75 & 1.61 \\
\hline 4 & 2.24 & 1.49 & 1.50 & 1.84 & 2.15 & 2.85 & 2.37 & 2.91 \\
\hline 5 & 1.94 & 3.31 & 1.03 & 2.61 & 1.99 & 1.67 & 2.13 & 1.71 \\
\hline 6 & 1.29 & 1.57 & 2.20 & 1.15 & 1.29 & 1.50 & 1.16 & 1.24 \\
\hline 7 & 2.49 & 1.07 & 3.64 & 2.50 & 2.41 & 1.76 & 2.43 & 1.90 \\
\hline 8 & 2.89 & 5.48 & 4.29 & 3.78 & 2.93 & 4.95 & 2.94 & 5.37 \\
\hline 9 & 1.92 & 0.93 & 1.99 & 1.05 & 1.86 & 1.62 & 1.85 & 1.81 \\
\hline 10 & 1.79 & 2.38 & 3.92 & 2.51 & 1.78 & 4.06 & 1.64 & 3.63 \\
\hline 11 & 3.23 & 3.87 & 6.28 & 4.29 & 3.26 & 2.59 & 3.37 & 2.63 \\
\hline 12 & 1.98 & 1.49 & 3.21 & 0.93 & 1.94 & 1.89 & 2.05 & 2.49 \\
\hline 13 & 2.93 & 4.46 & 3.29 & 4.03 & 2.91 & 4.18 & 2.69 & 3.99 \\
\hline 14 & 2.96 & 1.85 & 5.81 & 3.28 & 3.10 & 2.50 & 3.01 & 2.17 \\
\hline 15 & 2.20 & 3.32 & 2.73 & 1.36 & 2.20 & 2.73 & 2.32 & 2.79 \\
\hline 16 & 2.27 & 3.83 & 4.31 & 3.86 & 2.25 & 2.79 & 2.30 & 3.20 \\
\hline 17 & 2.23 & 2.04 & 5.76 & 1.29 & 2.25 & 1.18 & 2.25 & 1.24 \\
\hline 18 & 2.90 & 5.98 & 4.55 & 3.30 & 2.92 & 3.30 & 2.92 & 3.39 \\
\hline 19 & 1.97 & 2.17 & 4.43 & 2.79 & 2.00 & 2.97 & 2.31 & 2.79 \\
\hline 20 & 2.65 & 1.10 & 1.75 & 1.53 & 2.58 & 1.68 & 2.56 & 1.77 \\
\hline 21 & 2.30 & 5.62 & 6.04 & 3.28 & 2.31 & 2.51 & 2.25 & 2.49 \\
\hline 22 & 2.10 & 1.76 & 1.19 & 1.58 & 2.09 & 1.21 & 2.29 & 1.20 \\
\hline 23 & 2.80 & 2.47 & 1.82 & 2.87 & 2.82 & 4.76 & 2.61 & 4.90 \\
\hline 24 & 2.42 & 4.90 & 2.46 & 2.75 & 2.34 & 1.09 & 2.30 & 1.00 \\
\hline 25 & 2.08 & 1.68 & 2.61 & 1.73 & 2.08 & 3.02 & 2.33 & 3.13 \\
\hline 28 & 2.60 & 0.85 & 1.82 & 1.76 & 2.66 & 1.04 & 2.69 & 1.28 \\
\hline 29 & 2.39 & 2.87 & 3.30 & 2.82 & 2.34 & 3.24 & 2.39 & 3.62 \\
\hline 30 & 3.18 & 4.86 & 4.79 & 4.08 & 3.15 & 3.77 & 3.04 & 3.12 \\
\hline Dec 01 & 3.65 & 1.47 & 1.56 & 1.95 & 3.69 & 2.64 & 3.69 & 2.26 \\
\hline 2 & 2.59 & 4.74 & 5.29 & 3.79 & 2.56 & 3.74 & 2.80 & 4.31 \\
\hline 18 & 2.42 & 2.64 & 5.12 & 3.12 & 2.41 & 2.96 & 2.50 & 3.35 \\
\hline 29 & 2.85 & 5.48 & 5.03 & 4.22 & 2.88 & 4.41 & 2.91 & 4.45 \\
\hline Jan 06 & 2.01 & 4.17 & 4.99 & 3.28 & 1.98 & 3.55 & 1.76 & 3.52 \\
\hline 8 & 2.68 & 1.4 & 3.01 & 2.66 & 2.68 & 1.98 & 2.69 & 2.53 \\
\hline 9 & 2.02 & 3.91 & 3.23 & 2.97 & 2.00 & 3.60 & 2.00 & 3.60 \\
\hline 12 & 2.78 & 3.33 & 6.45 & 3.64 & 2.74 & 1.26 & 2.87 & 1.91 \\
\hline 13 & 2.20 & 2.01 & 1.26 & 0.96 & 2.18 & 3.02 & 2.01 & 2.92 \\
\hline 14 & 2.38 & 4.20 & 3.07 & 3.90 & 2.51 & 3.39 & 2.43 & 3.05 \\
\hline 15 & 2.57 & 1.56 & 1.40 & 2.15 & 2.53 & 1.61 & 2.77 & 1.66 \\
\hline 16 & 1.72 & 3.42 & 2.67 & 1.2 & 1.72 & 2.21 & 1.73 & 2.32 \\
\hline 18 & 2.03 & 2.36 & 2.14 & 0.87 & 1.96 & 0.70 & 2.11 & 0.73 \\
\hline 19 & 1.91 & 5.49 & 2.56 & 2.35 & 1.91 & 2.16 & 1.82 & 2.34 \\
\hline 20 & 2.10 & 1.70 & 0.78 & 2.53 & 2.05 & 2.53 & 2.08 & 2.18 \\
\hline 21 & 2.26 & 1.73 & 2.00 & 1.64 & 2.27 & 2.52 & 2.18 & 2.52 \\
\hline 22 & 1.87 & 3.35 & 4.44 & 2.55 & 1.88 & 1.80 & 2.05 & 1.93 \\
\hline 23 & 2.08 & 1.89 & 5.16 & 1.96 & 2.08 & 1.46 & 1.92 & 1.43 \\
\hline 24 & 2.52 & 1.85 & 1.14 & 2.09 & 2.45 & 3.18 & 1.87 & 3.23 \\
\hline 25 & 1.84 & 3.15 & 0.94 & 2.53 & 1.82 & 1.53 & 2.12 & 1.55 \\
\hline 26 & 1.69 & 1.69 & 1.76 & 1.35 & 1.73 & 2.01 & 1.56 & 1.79 \\
\hline Feb 05 & 1.91 & 4.26 & 5.07 & 3.65 & 1.87 & 2.69 & 1.95 & 3.12 \\
\hline
\end{tabular}


Table 7 (continued)

\begin{tabular}{lllllllll}
\hline Date & $\begin{array}{l}\text { HUST- } \\
\text { GR. } \\
\text { 2016S }\end{array}$ & ITU-GR.16 & $\begin{array}{l}\text { ITSG- } \\
\text { GR.2014S }\end{array}$ & $\begin{array}{l}\text { ITSG- } \\
\text { GR.2014K }\end{array}$ & TON.-GR.01 & EIG.-51C & EIG.-5S & EGM2008 \\
\hline 10 & 2.39 & 5.29 & 6.32 & 2.93 & 2.36 & 2.53 & 2.29 & 2.49 \\
Mean & 2.32 & 2.96 & 3.37 & 2.52 & 2.32 & 2.52 & 2.32 & 2.57 \\
Sigma & 0.46 & 1.47 & 1.69 & 0.98 & 0.47 & 1.02 & 0.48 & 1.02 \\
Min & 1.29 & 0.85 & 0.78 & 0.87 & 1.29 & 0.70 & 1.16 & 0.73 \\
Max & 3.65 & 5.98 & 6.45 & 4.29 & 3.69 & 4.95 & 3.69 & 5.37 \\
\hline
\end{tabular}

${ }^{a}$ Additional statistical parameters such as the mean, standard deviation, minimal and maximal RMS value for each gravity model are also presented. The BM have been included in these calculations. All presented statistics in meters

the number of estimated arcs per model is increased from 10 to 50 .

When analyzing the distribution of RMS values in Table 7, it is also possible to notice another division of the models into two characteristic groups. They differ in the dispersion of RMS values. The standard deviations in Table 7 are the measure of this dispersion. The models EIGEN-5S, HUST-GRACE2016S and TONGJI-GRACE01 have clearly smaller dispersion at the level of $0.5 \mathrm{~m}$, but for these models the best RMS values are always above $1 \mathrm{~m}$. The remaining models belong to the second group with clearly larger (by a factor of 2-3) standard deviations in the range from $0.98 \mathrm{~m}$ to $1.69 \mathrm{~m}$. In this case, the best RMS values are below $1 \mathrm{~m}$, reaching $0.70 \mathrm{~m}$. However, this group can be divided further into two subgroups coinciding with the two groups mentioned above; the first subgroup consisted of ITSG-GRACE2014K, EIGEN-51C and EGM2008 with the standard deviations at the level of $1 \mathrm{~m}$ and the second one that consists of ITU-GRACE16 and ITSG-GRACE2014S with the standard deviations around $1.5 \mathrm{~m}$. Therefore, the following pattern can be presented: the lower dispersion of RMS values about $0.5 \mathrm{~m}$ and somewhat larger minimum values of RMS up to $1.29 \mathrm{~m}$ (Table 7), and on the other hand the larger RMS dispersion from $0.98 \mathrm{~m}$ to $1.69 \mathrm{~m}$ and the lower minimum values of RMS from $0.70 \mathrm{~m}$ to $0.87 \mathrm{~m}$. Regarding the maximum RMS values, the following pattern can also be indicated: the smaller the dispersion (smaller standard deviation), the lower the maximum RMS value. This pattern can be seen more clearly than in the case of the RMS minimum value, it occurs for all three groups of models divided according to the standard deviation values.

Taking into account RMS differences and all estimated 1-day orbital arcs, the loss of accuracy on average $0.46 \mathrm{~m}$ when using the models EIGEN-5S, HUST-GRACE2016S and TONGJI-GRACE01 in the domain of minimal RMS values (Min RMS values, Table 7) is smaller than the accuracy loss on average $1.73 \mathrm{~m}$ in the domain of maximal RMS values (Max RMS values, Table 7) in the case of the use of the remaining gravity models.
The different RMS values for the particular days in Table 7, being the basis for formulating the above observations, reflect first of all differences in the performance of the tested gravity models and to a much lesser extent the differences in the accuracy of corresponding 1-day arcs of the reference orbit. This statement is justified by the analysis of the values of SLR residuals of the PSO RD reference orbit for the period from November 1, 2009, until mid-January 2010 presented by Bock et al. (2011). As already mentioned, the RMS of these residuals is below $2 \mathrm{~cm}$, while the maximum values in individual cases for single SLR stations reach up to $10 \mathrm{~cm}$. In one isolated case, in the middle of December 2009, a residual of about $20 \mathrm{~cm}$ appears for Greenbelt station (Bock et al. 2011).

\section{Summary and conclusions}

The long-wavelength parts of eight GRACE-related gravity models were investigated and compared with each other by the fit of estimated orbital arcs to the official reduceddynamic and kinematic orbits of the GOCE satellite. The Cartesian coordinates of both types of the orbit were observations in the dynamic orbit determination process using the least-squares adjustment. Different variants of estimated arcs, with three different arc lengths, ten and fifty arcs estimated for each gravity model and different options for additional background dynamic models - with and without these models in the estimation process, were obtained. As a measure of the fit of estimated arcs, the RMS difference between these arcs and the reference orbits was adopted. The results were obtained in the time span between November 02, 2009, and February 11, 2010.

Comparing the efficiency of using the background models, it is evident that for the increasing orbital arc, this efficiency decreases, for example, almost a double decrease for the 1-day arcs compared to the 90.2-min arcs. It is caused by increasing systematic errors due to the simplified parametrization of estimated orbital arcs. 
Inclusion in the calculations (with ten estimated 1-day arcs for each model) of the background models did not significantly affect the relative performance of the tested gravity models compared to the option without considering these (background) models.

The obtained results confirm the better fit to the reduceddynamic orbit than to the kinematic orbit, which may be connected with a greater smoothness of the reduced-dynamic orbit and, consequently, its greater accuracy.

Taking into account the short arcs of $45.2 \mathrm{~min}$ and $90.2 \mathrm{~min}$ (for the ten estimated arcs for each tested model), the performance differences of given gravity models were not resolved due to too small differences between the RMS values for the particular gravity models. These differences were under the accuracy level (about $2 \mathrm{~cm}$ ) of the PSO reduced-dynamic and kinematic reference orbits. Thus, the performance of given gravity models is equivalent in the aspect of estimation of several tens of minute arcs within the reference orbit accuracy.

For the 1-day arcs, both for the ten and the fifty estimated arcs per each gravity model, without and with the background models, the performance differences between the investigated gravity models are visible in the following two aspects:

(a) The differences of RMS of fit of estimated arcs for the tested gravity models reach the level of several meters, significantly exceeding the $2 \mathrm{~cm}$ threshold of the reference orbit accuracy.

(b) The analysis of particular RMS values (variant with fifty arcs for each gravity model with the background models) reveals the characteristic pattern of the performance of the gravity models. The gravity models EIGEN-5S, HUST-GRACE2016S and TONGJIGRACE01, with exactly the same smallest mean RMS values (for all 1-day arcs), have the slightly higher minimum RMS values (by a factor on average 1.6) with the smaller range of RMS variations compared to the smaller minimum RMS values for the remaining gravity models but in turn with the larger range of RMS variations. Consequently, for the three aforementioned models, the smaller maximum values of RMS are obtained, while in turn for the remaining models, i.e., for ITU-GRACE16, ITSG-GRACE2014S, ITSGGRACE2014K, EIGEN-51C and EGM2008, the maximum RMS values are higher (by a factor on average $1.5)$.

Considering the aspect (b), it can be concluded that the described effects in a certain sense are mutually compensating in the domain of minimum and maximum RMS values. So, the performance of these both groups of gravity models in this respect can be regarded as complementary.
However, taking into account the smallest mean RMS values and the smaller standard deviations of RMS values, the models of the first group, i.e., EIGEN-5S, HUSTGRACE2016S and TONGJI-GRACE01 should be highlighted. Due to the same mean RMS values and similar values of standard deviations, the performance of these models can be treated as equivalent. Considering the distribution of RMS values for the particular orbital arcs, these gravity models work stably and predictably, without showing such large changes in daily RMS values as in the case of other models. It is worth reminding here that the gravity model EIGEN-5S was the a priori model in the estimation process of the reduced-dynamic PSO (reference orbit) (Bock et al. 2011). In turn, another model from this group HUST-GRACE2016S is based on the longest-period GRACE data from all tested models. The length of this period is 13 years (Zhou et al. 2017).

Acknowledgements The author would like to thank ESA for providing the GOCE satellite orbit with accompanying auxiliary data and ICGEM/GFZ for obtaining the geopotential models. The author would also like to thank the anonymous reviewer whose suggestions helped to improve this paper.

Open Access This article is distributed under the terms of the Creative Commons Attribution 4.0 International License (http://creativeco mmons.org/licenses/by/4.0/), which permits unrestricted use, distribution, and reproduction in any medium, provided you give appropriate credit to the original author(s) and the source, provide a link to the Creative Commons license, and indicate if changes were made.

\section{References}

Baur O, Bock H, Höck E, Jäggi A, Krauss S, Mayer-Gürr T, Reubelt T, Siemes C, Zehentner N (2014) Comparison of GOCEGPS gravity fields derived by different approaches. J Geodesy 88(10):959-973. https://doi.org/10.1007/s00190-014-0736-6

Bezděk A, Sebera J, Klokočník J, Kostelecký J (2014) Gravity field models from kinematic orbits of CHAMP, GRACE and GOCE satellites. Adv Space Res 53(5):412-429. https://doi. org/10.1016/j.asr.2013.11.031

Bock H, Jäggi A, Meyer U, Visser P, van den Ijssel J, van Helleputte T, Heinze M, Hugentobler U (2011) GPS-derived orbits for the GOCE satellite. J Geodesy 85(11):807-818. https://doi. org/10.1007/s00190-011-0484-9

Bock H, Jäggi A, Beutler G, Meyer U (2014) GOCE: precise orbit determination for the entire mission. J Geodesy 88(11):10471060. https://doi.org/10.1007/s00190-014-0742-8

Bruinsma SL, Marty JC, Balmino G, Biancale R, Foerste C, Abrikosov O, Neumayer H (2010) GOCE gravity field recovery by means of the direct numerical method. In: ESA living planet symposium, 28 June-2 July 2010, Bergen, Norway

Carrion D, Vergos G, Albertella A, Barzaghi R, Tziavos IN, Grigoriadis VN (2015) Assessing the GOCE models accuracy in the Mediterranean area. In: Assessment of GOCE geopotential models, Newton's bull. 5, June 2015 
Casotto S, Gini F, Panzetta F, Bardella M (2013) Fully dynamic approach for GOCE precise orbit determination. B Geofis Teor Appl 54(4):367-384. https://doi.org/10.4430/bgta0108

Chen Q, Shen Y, Zhang X, Chen W, Hsu H (2015) TongjiGRACE01: a GRACE-only static gravity field model recovered from GRACE Level-1B data using modified short arc approach. Adv Space Res 56(5):941-951. https://doi.org/10.1016/j. asr.2015.05.034

Cheng M, Ries JC (2015) Evaluation of GOCE gravity models with SLR orbit tests. In: Assessment of GOCE geopotential models, Newton's bull. 5, June 2015

Drewes H (2012) International centre for global earth models (ICGEM). In: The geodesist's handbook 2012, J Geodesy 86(10):787-974. https://doi.org/10.1007/s00190-012-0584-1

Drożyner A (1995) Determination of orbits with Toruń Orbit Processor system. Adv Space Res 16(12):93-95. https://doi. org/10.1016/0273-1177(95)98788-p

ESA (2010), GOCE Level 2 product data handbook, European GOCE gravity consortium. In: ESA Tech. Note GO-MA-HPF-GS-0110, European Space Agency, Noordwijk

Eshagh M, Najafi-Alamdari M (2007) Perturbations in orbital elements of a low Earth orbiting satellite. J Earth Space Phys 33(1):1-12

Förste C, Flechtner F, Schmidt R, Stubenvoll R, Rothacher M, Kusche J, Neumayer H, Biancale R, Lemoine J-M, Barthelmes F, Bruinsma S, Koenig R, Meyer U (2008) EIGEN-GL05C—a new global combined high-resolution GRACE-based gravity field model of the GFZ-GRGS cooperation. Geophys Res Abstracts 10:EGU2008-A-03426

Förste C, Bruinsma SL, Flechtner F, Marty JC, Dahle C, Abrikosov O, Lemoine JM, Neumayer H, Barthelmes F, Biancale R, König R (2014) EIGEN-6C4 - the latest combined global gravity field model including GOCE data up to degree and order 1949 of GFZ potsdam and GRGS Toulouse. Geophys Res Abstracts 16:3707

Gruber Th, Visser PNAM, Ackermann Ch, Hosse M (2011) Validation of GOCE gravity field models by means of orbit residuals and geoid comparisons. J Geodesy 86:807-818. https://doi. org/10.1007/s00190-011-0484-9

Guo JY, Shang K, Jekeli C, Shum CK (2015) On the energy integral formulation of gravitational potential differences from satellite-tosatellite tracking. Celest Mech Dyn Astr 121(4):415-429

Hirt C, Rexer M, Claessens S (2015) Topographic evaluation of fifthgeneration GOCE gravity field models globally and regionally. In: Assessment of GOCE geopotential models, Newton's bull. 5, June 2015

Kvas A, Mayer-Gürr T, Zehentner N, Klinger B (2014) ITSGGrace2014 s: combined estimation of Earth's static and time variable gravity field. In: Geodätische Woche 2014, Berlin, Germany

Lejba P, Schillak S, Wnuk E (2007) Determination of orbits and SLR stations' coordinates on the basis of laser observations of the satellites Starlette and Stella. Adv Space Res 40(1):143-149. https:// doi.org/10.1016/j.asr.2007.01.067

Matos ACOC, Blitzkow D, Nascimento Guimarães G, Lobianco MCB, Oliveira Campos I (2015) Evaluation of recent GOCE geopotential models in South America. In: Assessment of GOCE geopotential models, Newton's bull. 5, June 2015

Mayer-Gürr T, Zehentner N, Klinger B, Kvas A (2014) ITSGGrace2014: a new GRACE gravity field release computed in Graz. In: GRACE Science team meeting, Potsdam, Germany, September 29 2014-October 12014

Melbourne W, Anderle R, Feissel M, King R, McCarthy D, Smith D, Tapley B, Vincente R (1983) Project MERIT standards. In: U.S. Naval Observatory Circulars 167, Washington, DC
Pavlis NK, Holmes SA, Kenyon SC, Factor JK (2012) The development and evaluation of the Earth Gravitational Model 2008 (EGM2008). J Geophys Res 117:B04406. https://doi. org/10.1029/2011jb008916

Reigber C, Jochmann H, Wünsch J, Petrovic S, Schwinzer P, Barthelmes F, Neumayer KH, König R, Förste C, Balmino G, Biancale R, Lemoine JM, Loyer S, Perosanz F (2005) Earth gravity field and seasonal variability from CHAMP. In: Earth observation with CHAMP - results from three years in orbit. Springer, Berlin, pp 25-30

Rummel R, Yi W, Stummer C (2011) GOCE gravitational gradiometry. J Geod 85:777-790. https://doi.org/10.1007/s00190-011-0500-0

Sośnica K, Thaller D, Jäggi A, Dach R, Beutler G (2012) Sensitivity of LAGEOS orbits to global gravity field models. Artif Sat 47(2):47-65. https://doi.org/10.2478/v10018-012-0013-y

Šprlák M, Gerlach C, Pettersen BR (2015) Validation of GOCE global gravitational field models in Norway. In: Assessment of GOCE geopotential models, Newton's bull. 5, June 2015

Standish EM, Newhall XX, Williams JG, Yeomans DK (1992) Orbital ephemerides of the sun, moon and planets. In: Seidelmann PK (ed) Explanatory supplement to the astronomical almanac. University Science Books, Mill Valley, pp 279-323

Strugarek D, Sośnica K, Jäggi A (2018) Characteristics of GOCE orbits based on satellite laser ranging. Adv Space Res 63:417-431. https ://doi.org/10.1016/j.asr.2018.08.033

Tapley B, Bettadpur S, Watkins M, Reigber C (2004) The gravity recovery and climate experiment: mission overview and early results. Geophys Res Lett 31:L09607. https://doi.org/10.1029/2004gl0199 20

Tsoulis D, Papanikolaou TD (2012) Numerical investigation of different gravity models in orbit propagation of two short CHAMP and GRACE-A Arcs. In: Sneeuw N, Novák P, Crespi M, Sansò F (eds) VII Hotine-Marussi symposium on mathematical geodesy. International Association of Geodesy Symposia, vol 137. Springer, Berlin, ISBN 978-3-642-22078-4, https://doi.org/10.1007/9783-642-22078-4_42

Tsoulis D, Papanikolaou TD (2013) Degree-wise validation of satellite-only and combined Earth gravity models in the frame of an orbit propagation scheme applied to a short GOCE arc. Acta Geod Geophys 48:305-316. https://doi.org/10.1007/s40328-013-0020-x

Tsoulis D, Papanikolaou TD (2014) Dynamic orbit parametrization and assessment in the frame of GOCE gravity models. Phys Earth Planet Inter 236:1-9. https://doi.org/10.1016/j.pepi.2014.08.003

Voigt C, Denker H (2015) Validation of GOCE gravity field models in Germany. In: Assessment of GOCE geopotential models, Newton's bull. 5, June 2015

Xu X, Zhao Y, Reubelt T, Tenzer R (2017) A GOCE only gravity model GOSG01S and the validation of GOCE related satellite gravity models. J Geod Geodyn 8(4):260-272. https://doi. org/10.1016/j.geog.2017.03.013

Yi W, Rummel R (2014) A comparison of GOCE gravitational models with EGM2008. J Geodyn 73:14-22. https://doi.org/10.1016/j. jog.2013.10.004

Zhou H, Luo Z, Zhou Z, Zhong B, Hsu H (2017) HUST-Grace2016 s: A new GRACE static gravity field model derived from a modified dynamic approach over a 13-year observation period. Adv Space Res 60(3):597-611. https://doi.org/10.1016/j.asr.2017.04.026 